Keywords: Interictal, Behavioral, Psychiatric

Symptoms, Personality Disorder.

\title{
The Temporolimbic Personality: A cross-national study
}

\author{
Ayse Aycicegi-Dinn* \\ Wayne M. Dinn ** \\ Catherine L. Caldwell-Harris ${ }^{\star \star}$ \\ * Istanbul University, Department of \\ Psychology, Istanbul, Turkey \\ ** Boston University, Department of \\ Psychology, Massachusetts, USA
}

\begin{abstract}
Background and objectives: Early investigators claimed that temporal lobe epilepsy (TLE) was associated with a personality traits and psychiatric symptoms collectively known as the interictal behavioral syndrome or Geschwind's syndrome. Interictal behavioral alterations associated with TLE included affective dysregulation; irritability and impulsive aggression; anxiety and obsessive-compulsive symptoms; paranoia; abnormal patterns of social interaction; schizophrenic-like symptoms and dissociative states; hypergraphia; and hyperreligiosity. A number of psychiatric disorders are known to have subclinical variants. Are recurrent temporolimbic seizure-like events (as determined by a self-report symptom inventory-the LSCL-Limbic System Checklist) among non-clinical subjects also associated with TLE-related psychiatric symptoms/ syndromes and personality features?

Methods: To test this, we examined the clinical/personality profiles of students who self-reported symptoms associated with temporolimbic seizures.

Results: In two separate studies, we found that American and Turkish students reporting temporolimbic seizure-like symptoms had clinical/personality profiles resembling interictal clinical/personality features.

Conclusions: Findings do not imply that high- or median-LSCL scorers are afflicted with an undiagnosed TL seizure disorder. Rather, the temporolimbic personality may be found, albeit in milder form, among individuals free of neurologic disease.
\end{abstract}

Received 27 July 2007

Revised 1 August 2008

Accepted 8 September 2008 


\section{Introduction}

Early investigators claimed that temporal lobe epilepsy (TLE) was associated with personality traits and psychiatric symptoms collectively known as the interictal (i.e., between seizure events) behavioral syndrome or Geschwind's syndrome ${ }^{1-3}$. Investigators reported that patients with TLE presented with psychiatric symptoms and personality features including affective dysregulation; irritability and impulsiveness; anxiety and obsessive-compulsive symptoms; paranoia; abnormal patterns of social interaction; grandiosity; schizophrenic-like symptoms and dissociative states; hypergraphia; and hyperreligiosity ${ }^{1-10}$. Geschwind and others focused on specific interictal behavioral phenomena including hypergraphia, hyperreligiosity, intense emotion, and unusual patterns of social interaction.

A number of more recent studies have confirmed that TLE is associated with a broad spectrum of neuropsychiatric symptoms/syndromes including: mood disorders; panic attacks; obsessive-compulsive symptoms; and schizophreniform disorders ${ }^{11-22}$. On the other hand, several studies lend support to the contention that the interictal behavioral syndrome associated with TLE does not represent a distinct diagnostic enti$\mathrm{ty}^{23,24}$.

\section{The temporolimbic personality}

Do non-clinical (i.e., student) subjects presenting with temporolimbic seizure-like events also demonstrate psychiatric symptoms/syndromes and personality features associated with TLE (i.e., the temporolimbic personality)? One intriguing possibility is that individuals presumably free of neurologic disease may present with psychiatric symptoms and personality traits that resemble behavioral characteristics associated with TLE, and that these personality/clinical features (the temporolimbic personality) will be associated with temporolimbic seizure-like events (as determined by a selfreport measure of simple and complex partial seizure symptoms). Persinger's ${ }^{25,26}$ work represents an early attempt to explore the possibility that complex-partial seizurelike "signs" among non-clinical subjects (i.e., university students) are associated with specific cognitive and emotional states. For example, Persinger ${ }^{25}$ found that university students obtaining high scores on a group of items from the Personal Philosophy Inventory assessing "Complex Partial Epileptic Signs" also obtained elevated scores on the Schizophrenia, Hypomania, and Psychasthenia subscales from the Minnesota Multiphasic Personality Inventory (MMPI) relative to low-scoring controls. Persinger administered items that reflect "classic ictal experiences." Persinger ${ }^{25}$ also noted that high-scoring subjects obtained elevated scores on the $F$ scale from the MMPI and suggested that this finding is "commensurate with the hypothesis that temporal lability predisposes the person to make unusual associations between events (and their response to them) given sensory-limbic hyperconnections..." (p. 1114). Persinger ${ }^{25}$ concluded that his "results support the existence of a continuum of temporal-lobe lability that extends into the normal population.... (and) people who are on the labile side of this continuum should have displayed most of the major themes of ictal and interictal experiences but without convulsions" (p. 1112). In a separate study, Persinger ${ }^{26}$ reported that university students obtaining elevated scores on a measure of "temporal lobe signs" were "more emotionally unstable, impulsive, emotionally sensitive... and tense (free-floating anxiety)." 
Describing his earlier work, Persinger ${ }^{25}$ observed that individuals obtaining high scores "on the various clusters of temporal lobe items from the inventory display significantly more compulsive thinking, affective anxiety, suspiciousness, and intellectual inefficiency as defined by the California Personality Inventory...” (p. 1112). One possibility is that temporolimbic structures, among individuals presenting with temporolimbic personality traits, may be unusually sensitive. Are temporolimbic personality traits associated with TL seizure-like symptoms in individuals presumably free of neurologic disease? This biological model of personality merits further study. To address this issue, we will determine whether temporolimbic seizurelike phenomena (as determined by a selfreport symptom inventory) among non-clinical (i.e., student) subjects are associated with TLE-related psychiatric symptoms/syndromes and personality features.

Bear $^{27}$ suggested that a "sensory-limbic hyperconnection" process might generate the affective/behavioral phenomena associated with the classic interictal syndrome in patients presenting with TLE. Schomer and Mesulam (Schomer, personal communication, May, 2006) have suggested that individuals may present with a clinical profile that resembles the interictal behavioral syndrome; however, there is no evidence of a pathologic process or temporolimbic seizure disorder. Schomer suggested that "hyperconnectivity" might generate "temporal lobe dysfunction" in the absence of unambiguous pathology (e.g., a temporolimbic seizure disorder). A number of psychiatric disorders are known to have subclinical variants ${ }^{28}$. We will explore the possibility that the temporolimbic personality is found, albeit in milder form, among individuals presumably free of neurologic disease.

Following Persinger, we compared the clinical/personality profiles of students obtaining elevated scores on a self-report measure of symptoms associated with temporolimbic seizures to the profiles of comparison subjects. Our study differs from Persinger's work ${ }^{25,26}$ in that we administered a clinical/personality test battery that included measures of Axis I (e.g., a structured diagnostic interview and clinical scales) and Axis II disorders as well as relevant personality questionnaires to university students living in distinct cultural environments (i.e., Western and Middle Eastern). In study I, we administered a test battery consisting of clinical measures and personality questionnaires to university students in the United States. A biological model of personality (e.g., the temporolimbic personality) would be strengthened if we were to observe similar response patterns among subjects living in discrete sociocultural settings. In a follow-up study, we administered a similar test battery to university students attending Istanbul University in the Republic of Turkey. Similar response profiles among research subjects residing in a discrete sociocultural milieu would lend support to the central thesis. 


\section{Study I}

\section{Method}

\section{Participants}

A clinical/personality test battery was administered to 124 students (37 male and 87 female) drawn from introductory psychology courses at Boston University. Students reporting a history of traumatic head injury (concussion with loss of consciousness) were excluded from analysis. Demographic data are presented in Table I. All participants completed the Limbic System Checklist-33 (LSCL) ${ }^{29}$. The Limbic System Checklist is a 33-question, self-administered symptom inventory developed by Teicher and colleagues ${ }^{29}$. Respondents indicated how frequently they have experienced symptoms associated with temporolimbic seizures including transient hallucinatory phenomena (e.g., visual and olfactory hallucinations), visceral symptoms associated with partial seizures (e.g., tachycardia), visual illusions and perceptual disturbances (e.g., macropsia and micropsia), altered states of consciousness and memory dysfunction (e.g., amnestic episodes), automatic movements, and other symptoms associated with simple and complex partial seizure states ${ }^{29}$.

\section{Data analysis plan}

American students obtaining LSCL scores that were $1 S D$ (or $>$ ) above the sample mean of $23.4(S D=12.8)$ were assigned to the high-scoring group (scores ranged from 36-59). Students obtaining LSCL scores that were at least $1 S D$ below the sample mean were assigned to the low-scoring group (scores ranged from 2-10). Final- ly, students obtaining LSCL scores that were within $1 S D(+/-)$ of the sample mean were assigned to the median-scoring group (scores ranged from 11-35) (see Table I). Analyses of variance (ANOVAs) and post hoc comparisons (Scheffe) were carried out. Fisher's Exact Test was used to determine if groups differed on categorical variables (i.e., DSM-IV axis-I diagnoses).

\section{Predictions}

We will determine whether students achieving elevated scores on a measure of partial and complex seizure-like symptoms (LSCL-33) demonstrate a clinical/personality profile that resembles descriptions of "interictal" psychiatric symptoms and personality features (i.e., the temporolimbic personality). Based on our review of the TLE literature and Persinger's ${ }^{25,26}$ work, we predict that subjects obtaining elevated scores on the LSCL-33 will obtain significantly higher scores, relative to comparison groups, on measures of behavioral disinhibition, depressive symptoms, obsessivecompulsive symptoms, social anxiety, magical ideation, and interpersonal dependence.

We also predict that individuals obtaining elevated scores on the LSCL-33 will achieve significantly higher scores on measures of the following personality disorders: schizotypal personality disorder (SPD); paranoid personality disorder (PPD); borderline personality disorder (BPD); narcissistic personality disorder (NPD); antisocial personality disorder (APD); dependent personality disorder (DPD); avoidant personality disorder (AvPD); and obsessive-compulsive personality disorder (OCPD). 
Table I

American sample: Mean (SD)

\begin{tabular}{|c|c|c|c|c|c|}
\hline & Low-LSCL & Median-LSCL & High-LSCL & $F$ & $P$ \\
\hline$n$ & 20 & 86 & 18 & & \\
\hline Age & $18.5(1.1)$ & $18.4(0.9)$ & $18.5(0.8)$ & 0.00 & 0.99 \\
\hline Education & $13.4(0.9)$ & $13.3(0.8)$ & $13.3(0.6)$ & 0.00 & 0.99 \\
\hline LSCL-Total & $7.0(2.4)^{*}$ & $22.3(6.7)^{*}$ & $47.1(6.7)^{*}$ & 201.1 & 0.001 \\
\hline \multicolumn{6}{|c|}{ Personality Diagnostic Questionnaire (PDQ-4) } \\
\hline Paranoid & $2.6(1.7)$ & $2.7(1.7)^{*}$ & $3.9(1.9)^{*}$ & 3.7 & 0.027 \\
\hline Schizoid & $1.0(1.5)$ & $0.9(1.2)$ & $1.4(1.3)$ & 1.0 & 0.352 \\
\hline Schizotypal & $1.4(1.5)^{*}$ & 2.3 (1.6)\# & $3.8(1.2)^{*} \#$ & 11.9 & 0.001 \\
\hline Histrionic & $2.1(1.8)$ & $2.3(1.6)$ & $3.3(1.9)$ & 2.9 & 0.054 \\
\hline Narcissistic & $2.9(1.5)$ & $3.0(1.8)$ & $3.8(2.0)$ & 1.5 & 0.216 \\
\hline Borderline & $1.3(1.3)^{*}$ & 2.0 (1.7)\# & $3.2(1.5)^{* \#}$ & 7.1 & 0.001 \\
\hline Antisocial & $1.0(0.8)^{*}$ & $1.2(1.2) \#$ & $2.0(1.2)^{* \#}$ & 4.3 & 0.015 \\
\hline Avoidant & $2.2(1.6) *$ & $2.2(1.8) \#$ & $4.1(2.3)^{*} \#$ & 7.8 & 0.001 \\
\hline Dependent & $1.0(1.3)^{*}$ & 1.5 (1.5)\# & $2.7(2.2)^{*} \#$ & 5.2 & 0.007 \\
\hline Obsessive-comp. & $3.3(1.8)$ & $3.9(1.6)$ & $4.3(2.0)$ & 1.8 & 0.169 \\
\hline \multicolumn{6}{|c|}{ Obsessive-compulsive inventory (OCI) ++ } \\
\hline Total score & $50.9(46.8)^{*}$ & $81.2(43.5)^{*}$ & $113.6(55.6)^{*}$ & 8.3 & 0.001 \\
\hline Checking & $11.2(11.7)^{*}$ & 14.9 (9.4)\# & $22.5(13.1)^{* \#}$ & 5.7 & 0.004 \\
\hline Doubting & $4.7(5.2)^{*}$ & 6.2 (4.8)\# & 10.1 (6.9)*\# & 5.3 & 0.006 \\
\hline Hoarding & $4.4(3.2)^{*} \#$ & $8.2(4.8) *$ & 10.3 (6.2)\# & 7.2 & 0.001 \\
\hline Neutralizing & $5.2(5.8)^{*}$ & $9.0(6.5)$ & $12.3(10.6)^{*}$ & 4.5 & 0.013 \\
\hline Obsessional & $6.3(9.8)^{* * \#}$ & $14.1(10.3)^{*}$ & 19.5 (13.0)\# & 7.1 & 0.001 \\
\hline Ordering & $8.4(7.4)^{*}$ & $12.1(8.4)$ & $16.6(8.5)^{*}$ & 4.3 & 0.015 \\
\hline Washing & $10.8(11.8)^{*}$ & $16.9(11.2)$ & $22.3(11.7)^{*}$ & 4.5 & 0.012 \\
\hline BDI & $5.5(4.5)$ & $8.1(7.8)$ & $9.5(5.6)$ & 1.6 & 0.199 \\
\hline Liebowitz++ & $28.6(21.0)$ & $36.9(19.5)$ & $43.7(20.7)$ & 2.7 & 0.067 \\
\hline
\end{tabular}

Frontal Lobe Personality Scale

\begin{tabular}{lcccrc}
\hline FLPS-Total & $87.6(12.7)^{*}$ & $95.5(16.1) \#$ & $106.6(17.4)^{* \#}$ & 6.8 & 0.001 \\
Disinhibition & $27.6(3.8)^{* \#}$ & $32.0(6.0)^{*}$ & $35.4(5.9) \#$ & 9.0 & 0.001 \\
Exec.Dysfunction & $36.0(6.4)^{*}$ & $38.4(7.1)^{*}$ & $43.1(9.0)^{* \#}$ & 4.7 & 0.011 \\
Apathy & $24.0(5.5)$ & $25.2(6.3)$ & $28.0(5.7)$ & 2.1 & 0.119 \\
\hline \multicolumn{7}{c}{ Schizotypal personality questionnaire-brief } \\
\hline Total & $5.5(4.2)^{*}$ & $8.2(4.1)^{*}$ & $13.4(3.5)^{*}$ & 18.4 & 0.001 \\
Cognitive & $2.1(1.5)^{*}$ & $3.3(2.0)^{*}$ & $5.6(1.7)^{*}$ & 17.1 & 0.001 \\
Interpersonal & $2.0(2.0)^{*}$ & $2.7(2.3) \#$ & $4.2(1.7)^{* \#}$ & 4.7 & 0.01 \\
Disorganization & $1.3(1.7)^{*}$ & $2.3(1.9) \#$ & $3.6(1.6)^{* \#}$ & 7.0 & 0.001 \\
Magical ideation Scale & $4.1(3.3)^{*}$ & $8.0(5.0)^{*}$ & $13.9(6.3)^{*}$ & 18.5 & 0.001 \\
\hline \multicolumn{7}{c}{$\mathrm{I}_{7}$ Impulsiveness questionnaire } \\
Venturesomeness & $8.2(3.7)$ & $8.8(3.9)$ & & \\
Empathy & $12.2(3.4)$ & $13.3(4.2)$ & $14.8(3.9)$ & 0.2 & 0.789 \\
Impulsivity & $5.8(4.3)^{*}$ & $6.6(4.4) \#$ & $9.7(4.5)^{* \#}$ & 4.3 & 0.119 \\
\hline
\end{tabular}

++ Note $=2$ subjects did not complete the OCI. 1 subject did not complete the LSAS.

* and \# symbols indicate Scheffe results. The* and \# symbols indicate that the groups differ significantly from each other.

LSCL = Limbic System Checklist-33; BDI = Beck Depression Inventory; Liebowitz $=$ Liebowitz Social Anxiety Scale. 
The aforementioned predictions are based on our review of the TLE literature which revealed that patients presenting with TLE may demonstrate the following symptoms: schizophreniform phenomena; paranoia; rapid mood shifts; interpersonal dependence; grandiosity; behavioral dyscontrol; fear of criticism; and obsessive-compulsive phenomena.

We also expect that individuals obtaining high scores on the LSCL will demonstrate elevated scores on measures of positive schizotypal symptoms; however, we predict that they will not obtain elevated scores on measures of negative schizotypal symptoms (e.g., interpersonal deficits and apathy). We also anticipate that individuals obtaining high scores on the LSCL will demonstrate elevated scores on measures of narcissistic and antisocial personality; however, we predict that subjects obtaining elevated scores on the LSCL will not present with psychopathic personality features (such as a lack of empathy and thrill-seeking behavior) that are associated with both NPD and APD. That is, although we expect to observe elevated scores on measures on narcissism and antisocial behavior (reflecting grandiosity and behavioral dyscontrol/ interpersonal conflict, respectively), we predict that individuals obtaining high scores on the LSCL will describe normal "empathic" responses, as determined by a self-report measure of empathy. Students obtaining high scores on the LSCL will not demonstrate significantly higher scores on a measure of venturesomeness. Note that we are predicting that students obtaining elevated LSCL scores will also achieve elevated scores on measures of avoidant and dependent personality disorders. This prediction reflects the observation that TLE patients presenting with interictal behavioral changes may form intense emotional attachments; however, they are hyper-responsive to criticism and fear abandonment, and may avoid establishing interpersonal relations (a classic approachavoidance conflict). In addition, we predict that a significantly greater number of subjects presenting with high LSCL scores will fulfill diagnostic criteria for the following Axis-I disorders: major depressive disorder; dysthymic disorder; panic disorder; generalized anxiety disorder; social anxiety disorder; and substance abuse disorders.

\section{Description of clinical/personality measures}

Mini International Neuropsychiatric Interview $\left(\right.$ MINI) ${ }^{30}$. The MINI is a structured diagnostic interview based on DSM$I V$ and $I C D-10$ criteria. We administered the following diagnostic modules:

- Major Depressive Episode (current and lifetime)

- Dysthymia (current: past 2 years)

- Panic Disorder (past month)

- Social Anxiety Disorder (past month)

- Alcohol Abuse/Dependence (past 12 months)

- Substance (non-alcohol) Abuse/Dependence (past 12 months)

- Generalized Anxiety Disorder (past 6 months)

Beck Depression Inventory $(B D I)^{31}$. The BDI is a self-administered instrument and was employed to assess the magnitude of depressive symptoms.

Liebowitz Social Anxiety Scale (LSAS $)^{32}$. The Liebowitz scale yields two subscale scores reflecting symptom severity and avoidance behavior. Subjects are instructed to indicate the degree of anxiety they experi- 
ence during specific social situations. Subjects also indicate how frequently they avoid these situations.

\section{Obsessive-Compulsive Inventory $(\text { OCI })^{33}$.} The OCI is a self-administered measure of the following obsessive-compulsive (OC) symptom subtypes (seven subscales) (note that the patient's score reflects both symptom frequency and the level of distress generated by the symptom): compulsive checking; compulsive doubting; hoarding; ordering; obsessional ideation; mental neutralizing; and washing rituals.

Personality Diagnostic Questionnaire-4 $(\boldsymbol{P D Q}-4)^{34}$. The Personality Diagnostic Questionnaire (PDQ-4) is a true-false, selfreport measure of Axis-II disorders including: schizotypal personality disorder; paranoid personality disorder; schizoid personality disorder; borderline personality disorder; narcissistic personality disorder; histrionic personality disorder; antisocial personality disorder; dependent personality disorder; avoidant personality disorder; and obsessive-compulsive personality disorder.

$I_{7}$ Impulsiveness Questionnaire ${ }^{35}$. The $\mathrm{I}_{7}$ Questionnaire is a forced-choice instrument. Respondents were asked to indicate whether they agree or disagree with a series of statements related to three personality dimensions: impulsiveness; venturesomeness; and empathy.

Schizotypal Personality Questionnaire$\boldsymbol{B}(\boldsymbol{S P Q}-\boldsymbol{B})^{36}$. This forced-choice, self-report instrument (based on the original 74-item SPQ) consists of 22 items that reflect three discrete clusters or factors including: Factor I) Cognitive or Perceptual Deficits (i.e., positive schizotypal symptoms); Factor II) Interpersonal Deficits (i.e., negative schizotypal symptoms); and Factor III) Disorganization (e.g., disorganized communication $)^{36}$.
Magical Ideation Scale (MIS) ${ }^{37}$. The Magical Ideation Scale is a forced-choice questionnaire. Respondents were asked to indicate whether they agree or disagree with statements related to schizophrenic-like phenomena (e.g., ideas of reference).

Frontal Lobe Personality Scale (FLPS $)^{38}$. Respondents were instructed to indicate how frequently they experience symptoms or exhibit behaviors associated with frontal lobe behavioral syndromes including 1) behavioral disinhibition, 2) executive control deficits, and 3) apathy.

\section{Results}

\section{Axis-I disorders}

As shown in Table I, group differences on a measure of classic OCD symptoms (OCI-total score) were highly significant $(p<0.001)$ and in the expected direction. Subscale scores and the results of post hoc comparisons are presented in Table I. Contrary to expectations, group differences on measures of depressive and social anxiety symptoms did not achieve significance $(p>0.067)$; however, group differences were in the expected direction. To determine if groups differed on categorical variables (i.e., DSM-IV axis-I diagnoses), students obtaining LSCL scores that were $1 S D$ (or $>$ ) above the sample mean were assigned to the high-scoring group. The remaining students were assigned to the comparison group. Analysis revealed that a significantly greater number of individuals in the high-LSCL scoring group met $D S M-I V$ diagnostic criteria for alcohol abuse ( $p<0.004$, Fisher's Exact Test), alcohol dependence $(p<0.001$, Fisher's Exact Test), and drug abuse ( $p<0.021$, Fisher's Exact Test). Contrary to expectation, group differences on the remaining MINI diagnostic 
modules (Axis-I disorders) did not achieve statistical significance $(p>0.096)$. As shown in Table I, group differences on measures of classic obsessive-compulsive symptoms were marked.

\section{Axis-II disorders}

Group differences on measures of schizotypal, avoidant, borderline, and dependent personality disorders were marked $(p<$ 0.007 ) and in the expected direction (see Table I). Group differences on measures of paranoid and antisocial personality disorders were marginally significant $(p<0.027)$. Post hoc comparisons are presented in Table I. Contrary to expectation, group differences on the narcissistic and obsessive-compulsive personality disorder subscales did not achieve significance $(p>0.169)$. As expected, group differences on measures of schizoid personality and apathy did not approach significance $(p>0.119)$.

\section{Personality measures}

Groups differed significantly on measures of disinhibition $(p<0.001)$, positive schizotypal personality features, and magical ideation $(p<0.001)$ and in the expected direction. Group differences on the impulsiveness questionnaire $(p<0.014)$ were marginally significant. Contrary to expectation, groups differed on a measure of negative schizotypy $(p<0.01)$. Groups also differed significantly on measures of executive dysfunction $(p<0.011)$, disorganization $(p<$ $0.001)$, and impulsiveness $(p<0.014)$. Elevated LSCL scores were associated with executive control deficits and impulsiveness. The results of post hoc comparisons are shown in Table I. Group differences on mea- sures of venturesomeness and empathy did not approach significance $(p>0.119)$. LSCL groups did not differ on age or educational level.

\section{Substance abuse and the temporolimbic personality}

Group differences on measures of substance abuse / dependence raise the possibility that the presence of psychiatric symptoms among students obtaining elevated LSCL scores may be associated with substance abuse, rather than temporolimbic "hyper-responsiveness" per se. To address this issue, we compared the clinical/personality profiles of high-LSCL scorers meeting criteria for alcohol abuse/dependence disorders to the profiles of high-LSCL scorers presenting without alcohol abuse/dependence disorder. Independent-samples $t$-tests were carried out. Groups did not differ on measures of depression ( $p=0.95)$, OCD ( $p>$ $0.12)$, disinhibition and impulsivity $(p>$ $0.19)$, social anxiety $(p=0.60)$, magical ideation $(p=0.32)$, empathy $(p=0.92)$, venturesomeness $(p=0.25)$, negative schizotypy $(p=0.59)$, executive dysfunction ( $p=$ $0.76)$, apathy ( $p=0.49)$, and Axis-II disorders including paranoid ( $p=0.20)$, schizotypal $(p=0.46)$, schizoid $(p=0.11)$, histrionic $(p=0.57)$, narcissistic $(p=0.34)$, borderline $(p=0.11)$, antisocial $(p=0.15)$, avoidant $(p=0.42)$, dependent $(p=0.11)$, and obsessive-compulsive $(p=0.22)$ personality disorders. Group differences on the SPQ-B subscale assessing positive schizotypal symptoms were marginally significant $(p<0.04)$. Comorbid substance abuse does not account for the clinical/personality profile of students obtaining high scores on the LSCL. 


\section{Study II}

\section{Method}

\section{Participants}

During the second study, a similar test battery was administered to 96 students (24 male and 72 female) drawn from psychology courses at Istanbul University. Demographic data are presented in Table II. Written informed consent was obtained from all subjects. In addition to the measures described above, a Turkish version of the SCID-II Patient Questionnaire (SCID-II PQ) ${ }^{39}$ was administered. The SCID-II PQ is a self-report measure based on DSM-IV diagnostic criteria for personality disorders. Turkish translations of the $\mathrm{MINI}^{40}$, SPQ-B ${ }^{41}$, and the $\mathrm{BDI}^{42}$ were used. The first author translated the OCI, LSCL, FLPS, PDQ-4, I Impulsiveness Questionnaire, and the Liebowitz scale into Turkish. A second bilingual clinical psychologist independently translated the Turkish version into English and the second author compared these translations to the original instruments. Discrepancies in meaning with the original English version were noted and the Turkish translation was adjusted. Note that one item from the Turkish version of the $I_{7}$ Impulsiveness Questionnaire was deleted prior to administration. The translator concluded that most Turkish respondents would not be familiar with the activity described in the item.

\section{Data analysis plan}

In study II, we employed the same grouping strategy. Turkish students obtaining LSCL scores that were $1 S D$ (or $>$ ) above the sample mean of $26.1(S D=15.0)$ were assigned to the high-scoring group (scores ranged from 41-77). Students obtaining LSCL scores that were at least $1 S D$ below the sample mean were assigned to the lowscoring group (scores ranged from 4-11). Students obtaining LSCL scores that were within $1 S D(+/-)$ of the sample mean were assigned to the median-scoring group (scores ranged from 12-40) (see Table II). Analyses of variance (ANOVAs) and post hoc comparisons (Scheffe) were carried out. Fisher's Exact Test was used to determine if groups differed on categorical variables (i.e., DSMIV Axis-I diagnoses).

\section{Results}

\section{Axis-I disorders}

As predicted, groups differed significantly on measures of classic OCD symptoms $(p<$ $0.001)$, depressive and social anxiety symptoms $(p<0.001)$, and in the expected direction. Post hoc analyses are shown in Table II. To determine if groups differed on DSM-IV axis-I diagnoses (based on the MINI), students obtaining LSCL scores that were $1 S D$ (or $>$ ) above the sample mean were assigned to the high-scoring group. The remaining students were assigned to the comparison group. Analysis (Fisher's Exact Test) revealed that a significantly greater number of individual in the high-LSCL scoring group met DSM-IV diagnostic criteria for alcohol abuse and dependence $(p<0.021)$, drug abuse $(p<$ $0.021)$, major depressive disorder $(p<0.001)$, and generalized anxiety disorder $(p<0.003)$. Group differences on the remaining MINI diagnostic modules (Axis-I disorders) did not achieve statistical significance $(p>0.065)$. 
Table II

Turkish sample: Mean (SD)

\begin{tabular}{lcccrr} 
& Low-LSCL & Median-LSCL & High-LSCL & $F$ & $P$ \\
\hline$n$ & 16 & 65 & 15 & & \\
Age & $21.1(2.9)^{*} \#$ & $19.5(1.6)^{*}$ & $18.6(1.4) \#$ & 7.7 & 0.001 \\
Education & $14.1(3.2)$ & $13.1(1.1)$ & $13.0(0.0)$ & 2.8 & 0.061 \\
LSCL-Total & $8.3(2.6)^{*}$ & $24.6(8.3)^{*}$ & $51.8(11.4)^{*}$ & 109.7 & 0.001 \\
\hline
\end{tabular}

Personality Diagnostic Questionnaire (PDQ-4)

\begin{tabular}{|c|c|c|c|c|c|}
\hline Paranoid & $1.6(1.5)^{*}$ & $3.7(1.8) *$ & $5.0(1.4)^{*}$ & 15.6 & 0.001 \\
\hline Schizoid & $0.9(1.4)^{*}$ & $1.9(1.3)^{*}$ & $1.7(1.4)$ & 3.4 & 0.036 \\
\hline Schizotypal & $1.4(1.0)^{*} \#$ & $3.2(1.6) *$ & 4.0 (1.1)\# & 12.4 & 0.001 \\
\hline Histrionic & $2.4(1.2)^{*}$ & $3.6(1.8)^{*}$ & $5.0(1.2)^{*}$ & 8.8 & 0.001 \\
\hline Narcissistic & $1.4(1.2)^{*}$ & $3.4(1.5)^{*}$ & $4.8(2.1)^{*}$ & 18.4 & 0.001 \\
\hline Borderline & $1.4(1.0)^{*}$ & $3.8(2.1)^{*}$ & $5.8(1.6) *$ & 20.5 & 0.001 \\
\hline Antisocial & $0.1(0.4)^{*}$ & $1.3(1.3)^{*}$ & $2.2(1.3)^{*}$ & 11.6 & 0.001 \\
\hline Avoidant & $1.4(1.3)^{* \# \#}$ & 2.9 (1.7)\# & $4.0(1.5)^{*}$ & 10.0 & 0.001 \\
\hline Dependent & $0.6(1.1)^{*}$ & $1.7(1.7)$ & $2.4(1.4)^{*}$ & 4.8 & 0.01 \\
\hline Obsessive-comp & $2.3(1.1)^{* \#}$ & $3.6(1.3) \#$ & $4.3(1.7)^{*}$ & 9.0 & 0.001 \\
\hline \multicolumn{6}{|c|}{ SCID-II PQ } \\
\hline Paranoid & $3.3(1.6) * \#$ & $4.6(1.5)^{*}$ & $5.6(1.4) \#$ & 8.4 & 0.001 \\
\hline Antisocial & $0.8(0.7)$ & $1.5(1.5)$ & $2.0(1.5)$ & 3.0 & 0.052 \\
\hline Avoidant & $1.7(1.6)^{*} \#$ & $3.0(1.6)^{*}$ & 3.6 (1.7)\# & 5.8 & 0.004 \\
\hline Borderline & $2.8(2.3)^{*}$ & $7.4(3.0)^{*}$ & $10.5(2.7)^{*}$ & 27.3 & 0.001 \\
\hline Dependent & $1.9(1.4)$ & $2.6(1.5)$ & $2.7(1.7)$ & 1.4 & 0.24 \\
\hline Histrionic & $4.5(2.2)^{*} \#$ & $6.3(1.8)^{*}$ & 6.9 (1.4)\# & 8.1 & 0.001 \\
\hline Narcissistic & $2.2(1.4)^{*}$ & $4.1(2.3) *$ & $5.8(2.2) *$ & 10.3 & 0.001 \\
\hline Obsessive-comp & $3.9(1.4) * \#$ & $6.2(1.8) *$ & 6.3 (2.7)\# & 9.8 & 0.001 \\
\hline Schizotypal & $2.0(1.3)^{*}$ & $3.3(1.9)^{*}$ & $5.1(1.9)^{*}$ & 11.3 & 0.001 \\
\hline Schizoid & $0.8(0.9)$ & $0.9(0.9)$ & $1.1(1.1)$ & 0.6 & 0.56 \\
\hline \multicolumn{6}{|c|}{ Obsessive-compulsive inventory (OCI) } \\
\hline Total score & $37.2(25.3) *$ & $96.9(43.9)^{*}$ & $147.7(56.3)^{*}$ & 23.7 & 0.001 \\
\hline Checking & $7.0(5.5)^{* \#}$ & $18.9(11.4)^{*}$ & 26.9 (17.5)\# & 11.0 & 0.001 \\
\hline Doubting & $2.6(3.2)^{*} \#$ & $7.7(5.5)^{*}$ & 9.8 (6.8)\# & 7.2 & 0.001 \\
\hline Hoarding & $3.1(2.8)^{*} \#$ & $7.1(4.2)^{*}$ & 9.3 (6.1)\# & 8.0 & 0.001 \\
\hline Neutralizing & $4.1(2.7)^{*}$ & $10.1(6.2)^{*}$ & $16.8(9.9)^{*}$ & 13.6 & 0.001 \\
\hline Obsessional & $8.1(9.7)^{*}$ & $20.0(11.9)^{*}$ & $34.0(13.8) *$ & 16.8 & 0.001 \\
\hline Ordering & $6.2(4.4)^{* \#}$ & $15.3(7.4)^{*}$ & $20.3(8.0) \#$ & 15.4 & 0.001 \\
\hline Washing & $5.8(4.4)^{*}$ & $17.5(10.8)^{*}$ & $30.3(12.9) *$ & 20.0 & 0.001 \\
\hline BDI & $3.7(4.6)^{*}$ & $9.3(6.7)^{*}$ & $17.2(9.8)^{*}$ & 14.3 & 0.001 \\
\hline Liebowitz & $12.0(7.7)^{*}$ & $21.0(10.6)^{*}$ & $30.5(10.4)^{*}$ & 12.7 & 0.001 \\
\hline \multicolumn{6}{|c|}{ Frontal Lobe Personality Scale } \\
\hline FLPS-Total & $75.7(13.0)^{*}$ & $90.1(13.3)^{*}$ & $101.6(15.6)^{*}$ & 14.0 & 0.001 \\
\hline Disinhibition & $20.8(3.2)^{*}$ & $27.7(4.9)^{*}$ & $31.6(6.5)^{*}$ & 18.9 & 0.001 \\
\hline Exec. Dysfunction & $31.1(6.3)^{*}$ & $37.2(6.7)^{*}$ & $43.3(7.8)^{*}$ & 12.2 & 0.001 \\
\hline Apathy & $23.7(6.5)$ & $25.1(5.4)$ & $26.7(6.3)$ & 1.0 & 0.37 \\
\hline
\end{tabular}

++ Note $=3$ subjects did not complete the OCI. 2 subjects did not complete the SPQ-B. 1 subject did not complete the I7 Questionnaire.

* and \# symbols indicate Scheffe results. The* and \# symbols indicate that the groups differ significantly from each other. LSCL = Limbic System Checklist-33; BDI = Beck Depression Inventory; Liebowitz = Liebowitz Social Anxiety Scale; SCID-II PQ = SCID-II Patient Questionnaire. 
Table II (continue)

\begin{tabular}{lccccc} 
& Low-LSCL & Median-LSCL & High-LSCL & $F$ & $P$ \\
\hline \multicolumn{7}{c}{ Schizotypal personality questionnaire-brief } \\
\hline Total & $7.8(4.4)^{*}$ & $10.8(3.4)^{*}$ & $12.4(4.1)^{*}$ & 6.2 & 0.003 \\
Cognitive & $3.0(1.4)^{*}$ & $4.4(1.6)^{*}$ & $5.5(1.6)^{*}$ & 8.4 & 0.001 \\
Interpersonal & $3.1(2.4)$ & $3.5(1.8)$ & $3.5(2.2)$ & 0.2 & 0.78 \\
Disorganization & $1.6(1.5)^{* \#}$ & $2.9(1.7)^{*}$ & $3.4(1.9) \#$ & 5.0 & 0.008 \\
\hline \multicolumn{7}{c}{$\mathrm{I}_{7}$ Impulsiveness questionnaire } \\
Venturesomeness & $8.4(3.5)$ & $9.7(3.0)$ & $10.2(2.7)$ & 1.5 & 0.23 \\
Empathy & $12.5(3.0)$ & $13.3(2.6)$ & $13.8(3.3)$ & 0.8 & 0.43 \\
Impulsivity & $5.9(4.4)$ & $7.5(3.8)$ & $9.3(4.5)$ & 2.7 & 0.07 \\
\hline
\end{tabular}

++ Note $=3$ subjects did not complete the OCI. 2 subjects did not complete the SPQ-B. 1 subject did not complete the I7 Questionnaire.

* and \# symbols indicate Scheffe results. The* and \# symbols indicate that the groups differ significantly from each other. $\mathrm{LSCL}=$ Limbic System Checklist-33; BDI = Beck Depression Inventory; Liebowitz = Liebowitz Social Anxiety Scale; SCID-II PQ = SCID-II Patient Questionnaire.

\section{Axis-II disorders}

As predicted, group differences on PDQ-4 subscales assessing paranoid, schizotypal, histrionic, narcissistic, borderline, antisocial, avoidant, and obsessive-compulsive personality disorders $(p<0.001)$, and SCID-II subscales assessing schizotypal, paranoid, histrionic, avoidant, narcissistic, borderline, and obsessive-compulsive personality disorders $(p<0.004)$ were highly significant and in the expected direction. Post hoc comparisons are presented in Table II. Group differences on the antisocial personality disorder subscale from the SCID-II PQ approached significance $(p=0.052)$. Group differences on the schizoid and dependent personality disorder subscales from the SCID-II did not approach statistical significance $(p>0.24)$.

\section{Personality measures}

Groups differed significantly on measures of disinhibition $(p<0.001)$ and executive dysfunction $(p<0.001)$, and positive schizotypal symptoms $(p<0.001)$. Post hoc com- parisons are shown in Table II. Group differences were in the expected direction. Groups did not differ on measures of negative schizotypal symptoms, apathy, venturesomeness, and empathy $(p>0.23)$. Group differences on the impulsiveness subscale approached, but did not reach, significance $(p<0.07)$. Group differences on the impulsiveness questionnaire were in the expected direction. Note that LSCL groups differed significantly on age $(p<0.001)$.

\section{Discussion}

Participants obtaining elevated scores on the LSCL demonstrate a clinical/ personality profile that resembles descriptions of TLErelated psychiatric symptoms and personality features. Findings support the "temporolimbic personality" hypothesis.

In study I, our findings indicate that temporolimbic seizure-like symptoms are associated with affective dysregulation, behavioral disinhibition, obsessional phenomena, 
substance abuse, schizotypal personality features, and maladaptive patterns of social interaction (e.g., borderline, avoidant, antisocial, dependent, and paranoid PD symptoms) in a high-functioning, non-clinical sample. As expected, groups did not differ on measures of empathy and venturesomeness. Contrary to expectation, group differences on measures of social anxiety, depression, narcissism, and obsessive-compulsive personality did not achieve statistical significance. However, group differences were in the expected direction.

In the second study, LSCL groups differed significantly on measures of classic OCD symptoms, disinhibition, and executive dysfunction. In addition, group differences on measures of depressive symptoms and social anxiety achieved statistical significance.

In both studies, temporolimbic seizurelike symptoms were associated with character pathology (as determined by PDQ-4 and SCID-II PQ scores). In study II, group differences on measures of personality disorder symptoms were striking. In both studies, participants obtaining high scores on the LSCL33 fulfilled diagnostic criteria for substance abuse disorders. Turkish subjects obtaining high scores on the LSCL-33 also met DSM$I V$ diagnostic criteria for major depressive disorder and generalized anxiety disorder.

As noted above, there were subtle differences in the clinical/personality profiles of American and Turkish subjects. Overall, the clinical/personality profiles of Turkish and American students were extraordinarily similar. As we observed in the introduction, a biological model of personality (e.g., the temporolimbic personality) would be strengthened if we were to observe similar response patterns among research participants living in distinct sociocultural environments. It is interesting to note that, in both studies, executive dysfunction and disorganization (i.e., scores on self-report measures) were associated with partial seizure-like symptoms. It is not surprising to find that subtle cognitive deficits and disorganized communication patterns are associated with temporolimbic seizure-like symptoms. Such regions play a crucial role in memory function. Moreover, temporolimbic structures receive projections from and project to prefrontal regions.

\section{Limitations}

One limitation of the present study is that we did not include measures of several classic interictal features such as hyperreligiosity, hypergraphia, changes in sexual activity (e.g., hyposexuality), and sleep disruption (Schomer, personal communication). Moreover, we have observed associations between scores on self-report measures of temporolimbic seizure-like phenomena and psychiatric symptoms/personality features. Investigators should determine whether temporolimbic seizure-like phenomena and TLE-related psychiatric/personality features are associated with variations in the structure and/or function (i.e., utilize neuroimaging technology) of temporolimbic regions among individuals free of central nervous system pathology. Of course, psychiatric symptoms (e.g., depressive symptoms or Cluster A personality disorders such as SPD) may disrupt cognitive functions. Indeed, there is extensive literature exploring the relation between schizotypal personality and executive control deficits.

\section{Conclusion}

American and Turkish students reporting temporolimbic seizure-like symptoms demon- 
strate clinical/personality profile that resembles descriptions of TLE-related psychiatric symptoms and personality features. Of course, we are not suggesting that high- or median-LSCL scorers are afflicted with an undiagnosed TL seizure disorder. A more plausible explanation is that variations in the function of temporolimbic structures are associated with partial seizure-like phenomena as well as psychiatric symptoms/personality features associated with TLE among individuals free of neurologic disease. This is consistent with Persinger's work. Indeed, our findings are consistent with Persinger's ${ }^{25}$ description of a "continuum of temporallobe lability" in the general population. As Persinger ${ }^{25}$ noted, "people who are on the labile side of this continuum should have displayed most of the major themes of ictal and interictal experiences but without convulsions" (p. 1112). Our findings suggest that temporolimbic hyper-responsiveness might generate partial seizure-like symptoms and behavioral/ emotional responses that resemble interictal phenomena among individuals free of CNS pathology. As Schomer observed, "hyperconnectivity" might generate "temporal lobe dysfunction" in the absence of neurologic disease (e.g., a temporolimbic seizure disorder).

\section{References}

1. Waxman SG, Geschwind N. Hypergraphia in temporal lobe epilepsy. Neurology 1974; 24: 629-636.

2. Waxman SG, Geschwind N. The interictal behavior syndrome of temporal lobe epilepsy. Arch Gen Psychiatry 1975; 32: 1580-1586.

3. Geschwind N. Behavioral changes in temporal lobe epilepsy. Psychol Med 1979; 9: 217-219.

4. Flor-Henry P. Schizophrenic-like reactions and affective psychoses associated with temporal lobe epilepsy: etiological factors. Am J Psychiatry 1969; 126: 148-152.
5. Blumer D. Temporal lobe epilepsy and its psychiatric significance. In: Benson DF, Blumer D, eds. Psychiatric aspects of neurologic disease. New York: Grune \& Stratton; 1975. p. 171-198.

6. Bear DM, Fedio P. Quantitative analysis of interictal behavior in temporal lobe epilepsy. Arch Neurol 1977; 34: 454-467.

7. Hermann BP, Riel P. Interictal personality and behavioral traits in temporal lobe and generalized epilepsy. Cortex 1981; 17: 125-128.

8. Bear D, Levin K, Blumer D, Chetham D, Ryder J. Interictal behaviour in hospitalised temporal lobe epileptics: relationship to idiopathic psychiatric syndromes. J Neurol Neurosurg Psychiatry 1982; 45: 481-488.

9. Bear DM, Freeman R, Greenberg M. Behavioral alterations in temporal lobe epilepsy. In: Blumer D, ed. Psychiatric aspects of epilepsy. Washington, DC: American Psychiatric Press; 1984. p. 197-227.

10. Blumer D. Evidence supporting the temporal lobe personality syndrome. Neurology 1999; 53(5 Suppl 2): S9S12.

11. Shukla GD, Srivastava ON, Katiyar BC, Joshi V, Mohan PK. Psychiatric manifestations in temporal lobe epilepsy: a controlled study. Br J Psychiatry 1979; 135: 411-417.

12. Sherwin I. The effect of the location of an epileptogenic lesion on the occurrence of psychosis in epilepsy. Adv Biol Psychiatry 1982; 8: 81-97.

13. Stoudemire A, Nelson A, Houpt JL. Interictal schizophrenia-like psychoses in temporal lobe epilepsy. Psychosomatics 1983; 24: 331-339.

14. Wall M, Tuchman M, Mielke D. Panic attacks and temporal lobe seizures associated with a right temporal lobe arteriovenous malformation: case report. J Clin Psychiatry 1985 ; 46: 143-145.

15. Gillig P, Sackellares JC, Greenberg HS. Right hemisphere partial complex seizures: mania, hallucinations, and speech disturbances during ictal events. Epilepsia 1988; 29: 26-29.

16. Carrieri PB, Provitera V, Iacovitti B, Iachetta C, Nappi C, Indaco A. Mood disorders in epilepsy. Acta Neurol 1993; 15: 62-67.

17. Kanner AM, Morris HH, Stagno S, Chelune G, Lunders H. Remission of an obsessive-compulsive disorder following a right temporal lobectomy. Neuropsychiatry Neuropsychol Behav Neurol 1993; 6: 126-129.

18. Kroll L, Drummond LM. Temporal lobe epilepsy and obsessive-compulsive symptoms. J Nerv Ment Dis 1993; 181: 457-458. 
19. Perini G, Tosin C, Carraro C, Bernasconi G. Interictal mood and personality disorders in temporal lobe epilepsy and juvenile myoclonic epilepsy. J Neurol Neurosurg Psychiatry 1996; 61: 601-605.

20. Altshuler L, Rausch R, Delrahim S, Kay J, Crandall P. Temporal lobe epilepsy, temporal lobectomy, and major depression. J Neuropsychiatry Clin Neurosci 1999; 11 : 436-443.

21. Meyer MA, Zimmerman AW, Miller CA. Temporal lobe epilepsy presenting as panic attacks: detection of interictal hypometabolism with positron emission tomography. J Neuroimaging 2000; 10: 120-122.

22. Monaco F, Cavanna A, Magli E, Barbagli D, Collimedaglia L, Cantello R, et al. Obsessionality, obsessivecompulsive disorder, and temporal lobe epilepsy. Epilepsy Behav 2005; 7: 491-496.

23. Mungas D. Interictal behavior abnormality in temporal lobe epilepsy. A specific syndrome or nonspecific psychopathology? Arch Gen Psychiatry 1982; 39: 108111.

24. Dodrill CB, Batzel LW. Interictal behavioral features of patients with epilepsy. Epilepsia 1986; 27(Suppl 2): S64-76.

25. Persinger MA. MMPI profiles of normal people who display frequent temporal-lobe signs. Percept Mot Skills 1987; 64: 1112-1114.

26. Persinger MA. Temporal lobe signs and personality characteristics. Percept Mot Skills 1988; 66: 49-50.

27. Bear DM. Temporal lobe epilepsy: a syndrome of sensory-limbic hyperconnection. Cortex 1979; 15: 357384.

28. Ratey J, Johnson C. Shadow syndromes. New York: Pantheon Books; 1997.

29. Teicher MH, Glod CA, Surrey J, Swett C. Early childhood abuse and limbic system ratings in adult psychiatric outpatients. J Neuropsychiatr Clin Neurosci 1993; 5: 301-306.

30. Sheehan D, Janavs J, Baker R, Harnett-Sheehan K, Knapp E, Sheehan M, et al. Mini International Neuropsychiatric Interview. J Clin Psychiatry 1998; 59(Suppl 20): 34-57.
31. Beck AT, Rush AJ, Shaw BF, Emery G. Cognitive therapy of depression. New York: Guilford; 1979.

32. Liebowitz MR. Liebowitz Social Anxiety Scale. J Clin Psychiatry 1999; 60(Suppl. 18): 65.

33. Foa E, Kozak M, Salkovskis P, Coles M, Amir N. The validation of a new obsessive-compulsive disorder scale: The Obsessive-Compulsive Inventory. Psychol Assess 1998; 10: 206-214.

34. Hyler SE. Personality Diagnostic Questionnaire (PDQ-4). New York: New York State Psychiatric Institute; 1994.

35. Eysenck S, Pearson P, Easting G, Allsopp J. Age norms for impulsiveness, venturesomeness, and empathy in adults. Pers Individ Dif 1985; 6: 613-619.

36. Raine A, Benishay D. The SPQ-B: A brief screening instrument for schizotypal personality disorder. J Personality Disorders 1995; 9: 346-355.

37. Eckblad M, Chapman LJ. Magical ideation as an indicator of schizotypy. J Consult Clin Psychol 1983; 51: 215-225.

38. Grace J, Malloy P. Frontal lobe personality scale. Providence, RI: Brown University; 1992.

39. Sorias S, Saygili R, Elbi H, et al. SCID II Hasta Formu. Bornova: Ege Universitesi; 1990.

40. Ornek T, Keskiner A. In: Sheehan D, Janavs J, Baker R, Harnett-Sheehan K, Knapp E, Sheehan M, et al. Mini International Neuropsychiatric Interview. J Clin Psychiatry 1998; 59(Suppl 20): 34-57.

41. Aycicegi A, Dinn WM, Harris CL. Validation of Turkish and English versions of the Schizotypal Personality Questionnaire-B. Eur J Psychol Assess 2005; 21(1): 34-43.

42. Hisli N. Depresyon Envanteri'nin gecerliligi uzerine bir calisma. Psikoloji Dergisi 1988; 6: 118-122.

Address for correspondence:

Wayne M. Dinn

42 Washington Terrace

Whitman, MA 02382

Tel: 781-447-6058

E-mail address: dinn@bu.edu 